\title{
Gabriel DANZIG, Apologizing for Socrates. How Plato and Xenophon created our Socrates
}

\author{
Louis-André Dorion
}

\section{(2) OpenEdition}

\section{Journals}

Édition électronique

URL : https://journals.openedition.org/philosant/954

DOI : 10.4000/philosant.954

ISSN : 2648-2789

\section{Éditeur}

Éditions Vrin

\section{Édition imprimée}

Date de publication : 1 novembre 2012

Pagination : 300-304

ISBN : 978-2-7574-0400-3

ISSN : $1634-4561$

Référence électronique

Louis-André Dorion, « Gabriel DANzIG, Apologizing for Socrates. How Plato and Xenophon created our Socrates », Philosophie antique [En ligne], 12 | 2012, mis en ligne le 01 novembre 2018, consulté le 03 décembre 2022. URL : http://journals.openedition.org/philosant/954 ; DOI : https://doi.org/10.4000/ philosant.954

\section{(c) $(1) \&$}

Creative Commons - Attribution - Pas d'Utilisation Commerciale - Pas de Modification 4.0 International - CC BY-NC-ND 4.0

https://creativecommons.org/licenses/by-nc-nd/4.0/ 
zione filosofica, cosicché la comunità scientifica dovrebbe stare ben attenta a non accantonare Saviesa grega arcaica solo per la scarsa familiarità di molti con la lingua catalana. Già così come è, l'opera ha meriti straordinari.

Livio ROSSETTI.

Enrique Hülsz Piccone, Nuevos ensayos sobre Heráclito : Actas del Segundo Symposium Heracliteum, Ciudad de México, Universidad Nacional Autónoma de México, 2009, ISBN 9786070212055.

Nuevos Ensayos Sobre Heráclito, pubblicato nel 2009 a cura di Enrique Hülsz Piccone, raccoglie gli atti del Secondo Symposium Eracliteum, tenutosi a Città del Messico nel giugno 2006. L'evento è importantissimo e, al tempo stesso, raro. Infatti, il primo Symposium si era tenuto a Chieti nel lontano 1981, giusto un quarto di secolo prima. Vero è che nel frattempo sono stati pubblicati, oltre a innumerevoli articoli e ai vari volumi della monumentale opera di Mouraviev, molti notevoli commenti e monografie, tra cui spiccano quelle di Conche, di Robinson, di Wilcox, di Dilcher, di Pradeau e lo stimolante libro di O'Connell ${ }^{1}$. Non si può quindi dire che l'argomento sia stato trascurato, ma lo stato frammentario in cui c'è giunto il libro di Eraclito lascia spazio per un'ampia gamma d'interpretazioni, ben rappresentata in questo Symposium.

Dopo una breve introduzione del curatore, gli atti iniziano con un testo di Serge Mouraviev dal titolo «Le livre d'Héraclite 2500 ans après. L'état actuel de sa reconstruction», che presenta il tanto atteso montaggio del testo eracliteo, composto da ben 199 elementi. Questa versione, preceduta da altre nel 1970, 1983 e 1991, non è ancora definitiva ma, nonostante sia già in corso di redazione la quinta versione, conserva ancora la sua validità, avendo già la struttura generale della ricostruzione finale ${ }^{2}$. I frammenti sono corredati da note che nell'insieme delineano $i$ criteri interpretativi dell'autore. L'importanza della memoria è tale da richiedere un'analisi approfondita, che non è possibile svolgere in questa sede senza rischiare di cadere in inaccettabili leggerezze. Una particolare menzione merita la parte introduttiva, in cui l'autore ripropone i criteri interpretativi esposti nella ben nota memoria del $1989^{3}$. Costatato che gli studi eraclitei non hanno condotto finora ad alcun vero successo per «l'assenza di qualsiasi metodo scientifico adeguato alla natura e allo stato dei testi», Mouraviev ritiene che la filologia dei testi di Eraclito debba cessare di essere un'arte, per diventare una scienza. A quest'obiettivo egli ha dedicato un'attività personale che si è articolata su un arco di almeno quattro decenni, con la pubblicazione dei suoi celebri Heraclitea. La ricostruzione del testo è coerente e i criteri guida sono ben documentati, ma è inevitabile che molte scelte

1. E. O'Connell, Heraclitus and Derrida. Presocratic Deconstruction, Peter Lang, New York, 2006.

2. La versione finale è appena uscita come Heraclitea IV: Refectio, A. Liber Heracliti, Academia Verlag, Sankt Augustin, 2011.

3. «Comment interpréter Héraclite (vers une méthodologie scientifique des études héraclitéennes)», Ionian Philosophy, a cura di K. J. Boudouris, Int. Ass. for Greek Philosophy, Athens, 1989, p. 271-279. 
rimangano congetturali, senza che ciò ne riduca in alcun modo il valore scientifico; valore che mi parrebbe però ingiusto negare ad altri autori che si sono dedicati allo studio di Eraclito con risultati di rilievo.

In «Representation and knowledge in a world of change », Daniel W. Graham sostiene che la dottrina del flusso è stata erroneamente attribuita a Eraclito, che è piuttosto un sostenitore della costanza nel mondo. Eraclito riconosce anche negli oggetti più usuali due aspetti contrastanti: qualcosa rimane la stessa e qualcosa differisce, ma agli uomini sfugge questa complessità. Per favorire la comprensione le spiegazioni teoriche sono inutili, ma è necessario, invece, introdurre il soggetto a esperienze vicarie, che imitino la struttura del mondo reale. In questo senso, Graham afferma, «Eraclito finisce per essere più un terapista che un epistemologo». Se il senso del discorso di Eraclito è mostrare la permanenza nel mutamento, mi sembra lecito chiedersi perché egli sia così pessimista riguardo alla capacità umana di comprendere. Gli uomini non sono per nulla turbati dal modificarsi degli oggetti e sono certamente in grado di riconoscere lo stesso fiume nel fluire delle acque.

Nel suo articolo «Heraclitus and logos - again», Thomas M. Robinson si dedica, in un linguaggio incisivo, a chiarire il significato della parola logos. Questo logos, che è vero sempre, è il logos di 'to sophon': un'entità divina, dotata della forza di un vincolo razionale «come anima del mondo, o forse universo in quanto razionale», eternamente impegnato a dichiarare che «tutte le cose sono uno». Robinson considera che l'unica seria alternativa a questa interpretazione sia la tesi, sostenuta da Martha Nussbaum, che il logos sia il pensiero dello stesso Eraclito, che con esso impone ordine a un mondo che cambia, tesi che però egli liquida piuttosto sbrigativamente, suggerendo che «suona più come Kant che come Eraclito». Diversi frammenti (in particolare 70, 78, 79, 83, 108) sottolineano però l'abisso che separa la conoscenza divina, to sophon, da quella umana: se il logos è questo discorso che ci trascende, perché, ci potremmo chiedere, Eraclito critica violentemente gli uomini, che non possono essere in grado di comprenderlo?

Nella memoria «Expresiones polares en Heráclito», Alberto Bernabé svolge un'accurata esegesi strutturale delle varie forme di espressioni polari riscontrabili nei frammenti di Eraclito, confrontandole con espressioni similari che compaiono nella tradizione letteraria da Omero fino ad autori del V secolo a.C., oltre che con passi delle laminette orfiche e del Carmen Aureum. Pur evidenziando la ricchezza delle risorse stilistiche di Eraclito, egli ritiene che l'uso delle espressioni polari non sia un fatto meramente stilistico: Eraclito vuol mostrare che la realtà risponde a un modello di logica oppositiva, e ricorre a paradossi linguistici per rivelare nel linguaggio la struttura della realtà. Questa interpretazione emerge però soltanto nelle conclusioni, rimanendo a mio avviso slegata dall'analisi svolta, e lascia degli interrogativi completamente aperti: cosa intende l'autore per logica oppositiva? In che senso la realtà obbedirebbe a tale logica?

La tesi che Francesc Casadesús Bordoy enuncia fin dall'inizio della sua memoria, «La transposición del vocabolario épico en el pensamiento filósofico de Heráclito», è che l'uso del linguaggio epico da parte di Eraclito è coerente con la sua concezione della struttura del cosmo come tensione di forze opposte che ne garantiscono la stabilità. Il linguaggio epico si accorda anche all'etica di Eraclito, secondo 
cui il premio per i morti in combattimento è essere onorati dagli dèi e dagli uomini. Eraclito disprezza la moltitudine, proprio come nell'Iliade i guerrieri aristocratici disprezzano la truppa anonima, paragonata a un gregge di pecore. Al coraggio del guerriero Eraclito associa anche la sapienza del saggio. Forse però l'autore identifica troppo l'etica eraclitea con quella epica. Lo scherno di Eraclito non sembra legato tanto all'origine sociale quanto all'effettivo comportamento della maggior parte degli uomini, indegno di cittadini della polis: la moltitudine è stigmatizzata per gli ideali mediocri, per la politica demenziale, per l'incapacità di comprendere alcune verità fondamentali, per l'ottusità e la creduloneria.

Un aspetto interessante dei frammenti di Eraclito è che in essi compaiono le più antiche testimonianze su altri pensatori, fatto salvo lo scherzoso riferimento di Senofane a Pitagora. A quest'argomento si riferiscono due delle memorie presentate al Symposium. Nel suo articolo «Heraclitus B 42: On Homer and Archilochus», Herbert Granger analizza il frammento in cui Eraclito critica aspramente i due poeti. L'autore ipotizza una serie di possibili ragioni della dura invettiva d'Eraclito: entrambi deplorano la natura comune e obiettiva della guerra; entrambi ignorano i principi più generali, come l'unità degli opposti; entrambi sono coinvolti nei misteri di Dioniso e di Demetra per gli inni sacri loro attribuiti.

Più rilevante per il pensiero di Pitagora che per quello di Eraclito è invece l'articolo di Carl Huffman, «La crítica de Heráclito a la investigación de Pitágoras en el fragmento 129». L'autore svolge un'interessante analisi filologica sull'uso dei

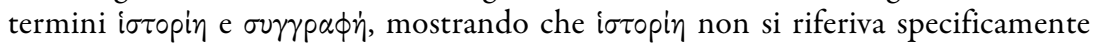
all'indagine scientifica, ma a qualsiasi tipo di ricerca; e che $\sigma v \gamma \gamma p a \phi \dot{\eta}$ non era usato soltanto per gli scritti in prosa, ma indicava qualsiasi registrazione scritta, sia in prosa, sia in versi, anche in testi molto corti come le registrazioni degli oracoli. Di

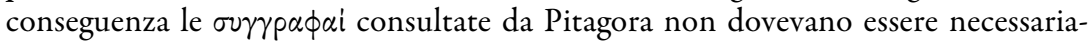
mente trattati ionici in prosa, come sostenuto da Kahn e da Riedweg, ma potrebbero aver incluso la poesia omerica ed esiodea. Notando, infine, che il verbo źk $\lambda \dot{\varepsilon} \gamma o-$ $\mu \alpha \iota$ si riferisce alle cose selezionate, piuttosto che quelle tra cui si opera la selezione, ritiene che con «questi scritti» ( $\tau \alpha \dot{\nu} \tau \alpha \varsigma \tau \dot{\alpha} \varsigma \sigma v \gamma \gamma p \alpha \phi \dot{\alpha} \varsigma)$ Eraclito intendesse proprio le compilazioni dei $\sigma \dot{\nu} \mu \beta 0 \lambda \alpha$ pitagorici che circolavano ai suoi tempi: collazioni raccogliticce, prive di originalità, mutuate dalla tradizione sapienziale antecedente.

La memoria di Omar D. Alvarez Salas «La 'teoria del flujo' de Heráclito a Epicarmo» si differenzia dalle altre perché incentrata su Epicarmo più che su Eraclito. Alvarez intende rivalutare il valore filosofico dei frammenti di Epicarmo, liberandolo dalla stretta associazione con Eraclito e restituendo così al comico siciliano l'originalità di pensiero che gli spetta. Egli ritiene che appartenga esclusivamente a

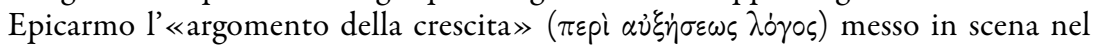
debitore che si rifiuta di pagare per il fatto di non essere più lo stesso uomo che aveva contratto il debito, essendosi a lui aggiunta nel frattempo qualche cosa e altra essendo venuta meno. È difficile però non riconoscere che l'argomento presenta molte affinità con quanto traspare, ad esempio, dai cosiddetti frammenti eraclitei del fiume, che cambia pur rimanendo sempre lo stesso fiume, proprio come l'individuo di Epicarmo può sostenere di essere cambiato, con effetto indubbiamente comico, ma è considerato lo stesso individuo dal suo creditore. 
L'intervento di Arnold Hermann «Parmenides versus Heraclitus?» è dichiaratamente una risposta all'articolo in cui Graham sostiene che Parmenide aveva effettivamente polemizzato con Eraclito ${ }^{4}$. Hermann ritiene che nessuno degli accostamenti citati da Graham sia significativo: Parmenide ha usato palintropos in circostanze completamente diverse da quelle di Eraclito; inoltre il termine non indica una contraddizione, ma ha il senso generale di «ritorno indietro». Interpretando la particella $\delta \dot{\varepsilon}$ in $28 \mathrm{~B} 6.9$ con senso fortemente avversativo, egli pensa infatti che palintropos non si riferisca al percorso dei mortali erranti, ma indichi invece un ritorno alla via della verità. Le argomentazioni di Hermann riguardano essenzialmente il pensiero di Parmenide, e benché personalmente non condivida la sua interpretazione del verso 28B6.9, la critica alla posizione di Graham mi sembra nel complesso condivisibile.

In «Acerca del significado del fragmento B62 (DK) de Heráclito», Beatriz Bossi affronta la lettura del frammento B62, esaminando in dettaglio diverse interpretazioni. Identificando nei «mortali» e negli «immortali» rispettivamente uomini e dèmoni custodi, l'autrice interpreta il frammento analizzando tutte le combinazioni dei quattro elementi in cui può essere decomposto: immortali, mortali, vivendo la morte di quelli, «avendo morto» la vita di quelli. Il frammento B62 è certamente molto denso nella sua sinteticità, e concordo con Schofield ${ }^{5}$ nel ritenerlo «un enigma che possa essere risolto soltanto da un lettore o una lettrice che abbia già meditato la sua via attraverso l'insieme del logos eracliteo». È verosimile che l'autrice abbia compiuto tale percorso, ma l'inquadramento nel contesto di un' interpretazione generale del pensiero eracliteo non è molto evidente nel testo.

Nella prima parte del suo articolo «The cosmic cycle, a playing child, and the rules of the game», Aryeh Finkelberg afferma che i testi provano la dottrina eraclitea della conflagrazione, e che quindi la teoria della stabilità cosmica è logicamente incompatibile con il pensiero di Eraclito. L'autore stabilisce dunque un parallelismo tra il posto occupato dall'anima nel ciclo microcosmico e quello occupato dal fuoco in quello macrocosmico, argomentando che il ciclo cosmico rappresenta l'incarnazione e poi la disincarnazione dell'anima divina, anima che s'incarna in quella umana e si libera con la morte. L'idea del corpo tomba dell'anima, formulata da Platone, risalirebbe così a Eraclito. Il fanciullo che gioca nel frammento 52 è una metafora del dio fiammeggiante, chiamato appunto «re» del gioco. Sebbene la dottrina eraclitea della conflagrazione non sia a mio avviso dimostrabile sulla sola base dei testi, la tesi di Finkelberg è svolta con indubbia coerenza e la conflagrazione è certamente in accordo con la sua interpretazione del pensiero d'Eraclito.

L'articolo di Livio Rossetti «Polymathia e unità del sapere in Eraclito: alle origini di una anomalia» si situa in posizione cardine per l'interpretazione gnoseologica di Eraclito. L'autore attribuisce alla coincidentia oppositorum - interdipen-

4. «Heraclitus and Parmenides», in Presocratic Philosophy. Essays in honour of Alexander Mourelatos, a cura di V. Caston e D. W. Graham, Ashgate, Burlington, 2002, p. $27-44$.

5. M. Schofield, «Heraclitus' Theory of Soul and its Antecedents», in S. Everson (ed.), Companions to Ancient Thought 2. Psychology, Cambridge, 1991, p. 13-34. 
denza o equalizzazione degli opposti, considerata idea centrale del pensiero dell' Efesio - un valore universale, e la definisce una reductio a unum dotata di un notevole «potenziale sistemico». Egli nota però che vi sono anche molti frammenti in cui Eraclito si limita ad affermare o a negare, a esprimere approvazione o disapprovazione, senza minimamente suggerire un possibile raccordo con enunciati complementari. Già tre decenni fa Rossetti ${ }^{6}$, nel corso del Symposium Heracliteum del 1981, concordava con Marcovich nel pensare che «uno dei principali difetti dell' interpretazione moderna d'Eraclito consiste nell'aver troppo creduto alla coerenza logica» e pensava che fosse necessario rassegnarsi all'idea che il testo contenga parti non congruenti con il principio sistemico. Ė senza dubbio lecito sostenere che Eraclito si contraddica, ma questa constatazione può anche costituire un potente stimolo per ricercare, a un diverso livello, una sintesi in grado di superare le aporie rilevate.

In «Flujo y Lógos. La imagen de Heráclito en el Cratilo y en el Teeteto de Platón», Enrique Hülsz Piccone ritiene che Platone abbia frainteso Eraclito attribuendogli una dottrina del «flusso universale», che nega l'effettiva possibilità della conoscenza. Egli avvicina il pensiero gnoseologico eracliteo a quello platonico, esplorando la possibilità che la teoria della «correttezza naturale dei nomi», enunciata da Cratilo nel dialogo omonimo, risalga effettivamente a Eraclito, che condividerebbe con Platone la tesi che il linguaggio incontri il suo fondamento epistemologico sul piano ontologico. Attribuire a Eraclito una versione del Flusso Universale implicherebbe, invece, un rifiuto della permanenza ontologica che sembra ben poco eracliteo. Sebbene sia innegabile che in diversi frammenti Eraclito esprima qualcosa che va ben oltre un banale riconoscimento del continuo modificarsi delle cose, riconoscere la permanenza ontologica di un ente soggetto a mutamento lascia aperta una questione importante: fino a che punto l'ente, pur mutando, rimane lo stesso e quando invece diventa altro? È possibile che Eraclito non abbia avvertito tale problema?

Nell'articolo «The limits of the soul: Heraclitus B45 DK. Its text and interpretation», Gábor Betegh svolge un'accurata analisi filologica del frammento 45. Egli ritiene che la parola $\pi \varepsilon i p \alpha \tau \alpha$ ( $\mathrm{i} \ll$ confini» dell'anima) debba essere conservata, perché la corruzione del termine nelle varie forme attestate è paleograficamente facile. Rifiuta invece il termine i $\dot{\omega} v$, introdotto da Diels, che non è attestato dai manoscritti. Sostiene poi persuasivamente che la lettura più attendibile del verbo

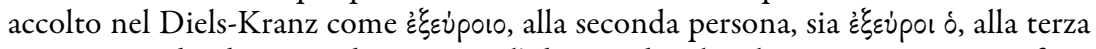
persona, rendendo così indeterminata l'identità di colui che ricerca. Ritiene, infine, grazie a diversi riferimenti indiretti, che l'ultima frase del frammento, oú $\tau \omega \beta \alpha \theta i v$ $\lambda$ joyov $^{\prime \prime} \chi \varepsilon l$, sia autentica. Leggendo $\psi \nu \chi \dot{y}$ come anima umana individuale e il logos come ragione profonda e penetrante dell'anima, interpreta il frammento nel senso che sia proprio chi percorre ogni sentiero colui che non trova i confini dell'anima.

L'articolo di Catherine Osborne, «"If all things were to turn to smoke, it'd be the nostrils would tell them apart" or Heraclitus on the pleasure of smoking»,

6. «Eraclito (e Solone) sulla Stasis», Atti del Symposium Heracliteum 1981, a cura di L. Rossetti, Roma, Edizioni dell'Ateneo, 1983, vol. 1, p. 347-359. 
prende in esame la testimonianza di Aristotele su Eraclito. Osborne mostra che, mentre è usuale fare riferimento alla percezione aristotelica degli aspetti cosmologici e metafisici di Eraclito, vi sono altre testimonianze disperse nel corpus aristotelico che vertono sull'anima, sul fumo, sui vapori, sui piaceri, considerate in genere isolatamente, ma che sono in realtà correlate. Aristotele è invocato dunque come testimone, forse non del tutto consapevole, di un tema fondamentale di Eraclito. Analizzando il frammento 67, l'autrice arriva a formulare un'interpretazione del pensiero gnoseologico eracliteo che mi pare condivisibile: «noi percepiamo con un occhio interessato [...] il mondo non è lo stesso per tutti perché lo approcciamo con preferenze e interessi distinti.» Se il mondo fosse fumo, ci interesserebbero solo gli odori, e se possedessimo soltanto l'olfatto, il mondo sarebbe per noi ben diverso da quello che ci appare. Osborne identifica dunque un importante tema eracliteo: «quello che è obiettivamente un mondo comune può risultare mondi assolutamente differenti per differenti abitanti.»

In «The fate of Heraclitus' book in Later Antiquity», David Sider si propone di scoprire se anche alcuni degli autori più tardi, oltre a Platone, Aristotele e Teofrasto, abbiano avuto accesso diretto al testo di Eraclito. Egli giunge alla conclusione che nessuna altra fonte abbia letto Eraclito in originale. Per esemplificare l'effetto distorcente delle successive interpretazioni e ricopiature del libro, prende in esame il frammento 22B124, il cui testo originale dovrebbe ridursi a non più di

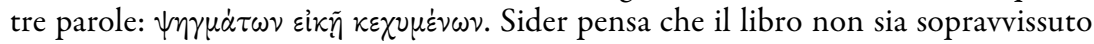
fino alla tarda antichità nella stesura originale, ma solo sotto forma di una raccolta di citazioni di seconda mano. Per questo motivo «possiamo giudicare l'autenticità di un frammento solo in base ai suoi meriti, quale che sia il modo che scegliamo per farlo».

Questo convegno rappresenta un momento importante degli studi su Eraclito, sia per il contributo fondamentale di Mouraviev, di cui si attendono gli sviluppi, sia perché vi si confrontano diverse tendenze interpretative su temi fondamentali del pensiero eracliteo. Mi fermerò brevemente su un solo tema, che ritengo centrale: diverse memorie interpretano la dottrina degli opposti come un equilibrarsi di tensioni che garantisce la stabilità. D'altra parte Rossetti mostra che attribuendo un valore sistemico alla coincidentia oppositorum è necessario riconoscere che Eraclito cade in contraddizioni. Si potrebbe allora pensare che l'uso eracliteo di termini opposti sia soltanto un motivo letterario, destinato a rendere evidente l'unità del tutto, piuttosto che l'enunciazione della natura dialettica della realtà. Osborne interpreta nel senso che le distinzioni sono opera del soggetto, anche se ammette che «là fuori» esistono sicuramente delle differenze da discernere. Forse, però, Eraclito dice proprio che «là fuori» è tutto uno, e che le differenze dipendono solo dai punti di vista.

Guido CALENDA.

Gabriel DANZIG, Apologizing for Socrates. How Plato and Xenophon created our Socrates, Lanham (Md.), Lexington Books, 2010, 280 p. ISBN 978-0-7391-3244-9

Cet ouvrage rassemble six études, dont trois (les chapitres 1,2 et 6 ; voir p. 14) sont des versions révisées d'articles parus antérieurement dans divers périodiques. Il 